Abanico Agroforestal. Enero-Diciembre 2020; 2:1-13. http://dx.doi.org/10.37114/abaagrof/2020.6 Artículo Original. Recibido: 16/12/2019. Aceptado: 10/04/2020. Publicado: 23/04/2020.

\title{
Respuesta productiva de maíces subtropicales y tropicales con fines forrajeros en una región semiárida
}

Productive response of subtropical and tropical corns for forage purposes in a semi-arid region

\section{Rivas-Jacobo Marco ${ }^{1} \stackrel{\mathrm{ID}}{ }$, Ballesteros-Rodea Gilberto ${ }^{1} \stackrel{\mathrm{ID}}{ }$, Lepe-Aguilar Rosa ${ }^{2} \underline{\mathrm{ID}}$, Zaragoza-Bastida Adrian ${ }^{3} \underline{\mathrm{ID}}$, Ibarra-Gudiño César ${ }^{2} \underline{\text { ID }}$, Rivero-Pérez Nallely 3 므.}

${ }^{1}$ Facultad de Agronomía y Veterinaria, Universidad Autónoma de San Luis Potosí, San Luis Potosí, México. ${ }^{2}$ Facultad de Medicina Veterinaria y Zootecnia de la Universidad Autónoma de Nayarit, Nayarit, México. ${ }^{3}$ Área Académica de Medicina Veterinaria y Zootecnia, Instituto de Ciencias Agropecuarias, Universidad Autónoma del Estado de Hidalgo, Hidalgo, México. *Autor de correspondencia: Ballesteros-Rodea Gilberto, gilberto.ballesteros@uaslp.mx, marco.rivas@uaslp.mx, isela.aguilar@uan.edu.mx, adrian_zaragoza@uaeh.edu.mx, cesaroctavio76@hotmail.com,nallely_rivero@uaeh.edu.mx.

\section{RESUMEN}

Se estimó el rendimiento de materia seca y de sus componentes morfológicos y de algunos caracteres de las plantas de maíz en la Facultad de Agronomía y Veterinaria de la Universidad Autónoma de San Luis Potosí, a $22.23^{\circ} \mathrm{LN}$ y a $100.85^{\circ} \mathrm{LO}$, a 1,835 m.s.n.m. en clima seco templado. Se utilizaron 26 genotipos de maíces, bajo riego en Primavera-Verano. La siembra se realizó en parcelas de 5 surcos de $0.90 \mathrm{~cm}$ de ancho $\times 5$ metros de largo. Se depositó una semilla cada $12 \mathrm{~cm}$ a una profundidad de $7 \mathrm{~cm}$. Se fertilizó con 160-40-00 (N-P-K). Se marcaron 10 plantas al azar en cada parcela. La cosecha se realizó en elote a $1 / 2$ de la línea de leche. Se midió el rendimiento de materia seca (RMS), de planta (RMSP), elote (RMSE), relación Elote:Planta completa (RELPC) y Planta:Planta completa (RPPC), Número de hojas por planta (NHOJAS), y de elote (NELOTES), Altura de planta (AP), Diámetro de tallo (DT). El mejor RMSP lo mostró OjitalCT $\left(17.8\right.$ t ha $\left.^{-1}\right)$ y Tampiqueño1 $\left(17.0 \mathrm{t} \mathrm{ha}^{-1}\right)$. TlanchiHgoA2 mostró el mayor valor de RMSE $\left(12.7 \mathrm{t} \mathrm{ha}^{-1}\right)$ y para RMS (28.6 t ha-1), lo que hace que estos genotipos sean viables para zonas semiáridas.

Palabras clave: Maíz, rendimiento, materia seca, componentes morfológicos y criollos

\begin{abstract}
The yield of dry matter and its morphological components and some characters of the corn plants in the Faculty of Agronomy and Veterinary of the Autonomous University of San Luis Potosí, at $22.23^{\circ} \mathrm{LN}$ and at $100.85^{\circ} \mathrm{LO}$, at $1,835 \mathrm{~m}$.a.s.I. in mild dry weather. 26 corn genotypes were used, under irrigation in SpringSummer. The sowing was carried out in plots of 5 rows of $0.90 \mathrm{~cm}$ wide $\times 5$ meters long. A seed was deposited every $12 \mathrm{~cm}$ at a depth of $7 \mathrm{~cm}$. It was fertilized with 160-40-00 (N-P-K). 10 plants were randomly marked on each plot. The harvest was done in corn at $1 / 2$ of the milk line. 10 plants were randomly marked on each plot. The harvest was done in corn at $1 / 2$ of the milk line. The yield of dry matter (YDM), plant (YDMP), corn (YDMC), ratio corn:Complete plant (RCCP) and Plant:Complete plant (RPCP), Number of leaves per plant (NLEAVES), and of corn (NCORN), Plant height (PH), Stem diameter (SD). The best YDMP was shown by OjitalCT (17.8 t ha-1) and Tampiqueño1 (17.0 t ha-1). TlanchiHgoA2 showed the highest value of YMDC (12.7 t ha-1) and for YDM (28.6 t ha-1), which makes these genotypes viable for semi-arid areas.
\end{abstract}

Keywords: Corn, yield, dry matter, morphological components and Creoles.

\section{INTRODUCCIÓN}

La producción de maíz forrajero en México es de gran importancia, debido a que representa una fuente de alimento energética básica e importante para la producción creciente de ganado lechero, y se estima, que de 2010 a 2018 pasó de 2,374,623 a 
2,529,672 cabezas, lo que representa un incremento de 155,049 cabezas (6.5\%); Jalisco (14\%), Durango (11.8\%), Chihuahua (11.4\%), Coahuila (9.5\%), Guanajuato (7.8\%), Hidalgo (7.8\%), Puebla (7.0\%), Querétaro (4.6\%) y México (4.1\%) (SIAP, 2020a). Aspecto que hace importante la búsqueda de alternativas de alimentación de calidad y de menor precio, para abaratar los costos de alimentación y hacer más rentable la industria lechera; ya que en las cuencas lecheras de México, el ensilado de maíz se utiliza comúnmente en la alimentación del ganado lechero, y llega a constituir del 30 al $40 \%$ de la ración en base seca en vacas en producción (González et al., 2005).

Dentro de los forrajes que más se consumen y producen en la comarca lagunera, región lechera, es el maíz; siendo el segundo cultivo de importancia, después de la alfalfa (González-Salas et al., 2018). Por otro lado, el constante crecimiento del número de cabezas de ganado en la comarca lagunera, demanda el aumento en la producción de maíz forrajero y plantea la necesidad de identificar materiales con buenas características forrajeras, por lo que entre los criterios de selección para identificar híbridos adecuados, está el que presenten alto rendimiento de grano, productividad y alta calidad de forraje (Sánchez et al., 2011).

Por otro lado, la producción de maíz forrajero en México ha tenido pocos avances, ya que la industria semillera no ha puesto a disponibilidad de los productores una amplia diversidad de variedades de maíz forrajero, que muestren mayor potencial productivo para mejorar los rendimientos de forraje verde para ensilar. Se tienen datos que la superficie sembrada en México para 2018 fue de 603,326 ha., con un rendimiento promedio de 29 ton/ha de forraje verde; y para el año de 2010 se tuvo una superficie sembrada de 535,621 ha., con un rendimiento promedio de 24 ton/ha de forraje verde (SIAP, 2020b); lo que demuestra que se ha tenido un incremento de 67,705 ha. (12.6\%), pero el rendimiento aún es bajo, a pesar de haber tenido un aumento de 5 ton/ha (20.8\%). El cultivo de maíz para ensilado en México presenta bajo rendimiento de materia seca por hectárea, bajo contenido de grano y alto de fibra, lo que ocasiona que la digestibilidad y energía del forraje sean bajas. Esta situación se debe en parte al uso de híbridos considerados como forrajeros, de elevada altura y gran capacidad para producir forraje, pero con pobre valor nutritivo; ya que por lo general esos genotipos se siembran a altas densidades de población, lo que ocasiona una escasa cantidad de grano (Núñez et al., 2005); además de que su formación no fue para maíces forrajeros (Peña et al., 2004).

En la búsqueda de mejorar los rendimientos y calidad del maíz forrajero, se han realizado diversos estudios para evaluar diversas variedades mejoradas y criollos de algunas regiones, con el fin de dar alternativas para mejorar los rendimientos y ofrecer genotipos de maíz que ofrezcan mayor rentabilidad y calidad forrajera. Entre ellos, ZaragozaEsparza et al. (2019) evaluaron 12 genotipos en Valles Altos, y observaron que los mayores rendimientos de materia seca fueron para los híbridos Búho, 501 x 497, 504 x 408, Puma 1163, H-48, 501 x 555 y 501 x 410 con 26.4, 25.6, 25.5, 25.4, 24.7, 24.7 y 
24.3 t ha $^{-1}$ de materia seca, respectivamente; y $74.0,64.1,68.8,75.0,69.4,65.8$ y 65.8 ton/ha de forraje verde, respectivamente.

La altura de planta de los genotipos estudiados fue variable, y mayor para Puma 1167, Puma 1163, H-48, 504 x 408 y H-51 AE con 2.4, 2.3, 2.2, 2.2 y 2.2 m, respectivamente. Lo que pone de manifiesto que existen en el mercado variedades alternativas para los productores que no pueden acceder a las variedades ofrecidas en el mercado por empresas internacionales. Sánchez et al. (2019) evaluaron seis híbridos comerciales en una región tropical, y observaron diferencias entre genotipos y que la altura varió de 203.1 a $167.2 \mathrm{~cm}$. El número de hojas fue de 10.7 a 9.6 hojas planta-1 $^{-1}$, el número de elotes planta $^{-1}$ de 1.26 a 1.0, el diámetro de tallo 5.7 a $4.8 \mathrm{~cm}$, el Peso de hojas planta-1 fue entre 162.3 a $122.9 \mathrm{~g}$; el peso de tallo estuvo entre 130.8 a 81.4 , rendimiento de forraje de 38.8 a 30.7 ton ha ${ }^{-1}$. Al igual la densidad de población influyó en el rendimiento, ya que a una densidad mayor y que fue de 83,333 plantas ha-1., mostró el mayor rendimiento de forraje con 41.8 ton ha $^{-1}$.

Otro estudio en maíces trilineales evaluados en una región tropical seca por Rivas et al. (2018), observaron variabilidad en los parámetros estudiados, y los mejores genotipos mostraron RMS de 32.8 a $27.6 \mathrm{t} \mathrm{ha}^{-1}$, para hoja de 5.7 a $5.0 \mathrm{t} \mathrm{ha}^{-1}$, para tallo 19.1 a 13.6 $\mathrm{t}$ ha ${ }^{-1}$, para elote 8.9 a $8.5 \mathrm{t} \mathrm{ha}^{-1}$; y el porcentaje de los componentes morfológicos fue para hoja de 16 a $22 \%$, elote 26 a $35 \%$ y tallo 43 a $58 \%$; aspectos que van a influir en el rendimiento total de la materia seca de los genotipos y de su calidad.

Por lo que el objetivo del presente estudio fue estimar el rendimiento de materia seca, componentes morfológicos y algunos caracteres de las plantas de 26 genotipos de maíz, originarios de regiones subtropical y tropical, bajo condiciones de un clima climática seco.

\section{Localización}

\section{MATERIAL Y MÉTODOS}

La investigación se realizó en la Facultad de Agronomía y Veterinaria de la Universidad Autónoma de San Luis Potosí, ubicada en el Ejido Palma de la Cruz, municipio de Soledad de Graciano Sánchez, S. L. P., en el Km. 14.5 de la Carretera San Luis PotosíMatehuala, que se ubica a $22^{\circ} 13^{\prime} 39.8^{\prime \prime}$ de Latitud Norte y a $100^{\circ} 50^{\prime} 58.3^{\prime \prime}$ de Longitud Oeste, a 1,835 m.s.n.m. El clima es seco templado; con temperatura media anual de 17.1 ${ }^{\circ} \mathrm{C}$. La precipitación pluvial es de 362 mm (García, 2004).

\section{Material genético y tratamientos}

Se utilizaron 23 genotipos de maíces locales, colectados en regiones subtropicales y tropicales de México (Nayarit, Veracruz e Hidalgo), dos genotipos locales y uno mejorado de la región semiárida en estudio, para compararlos; los cuales funcionaron como tratamientos (cuadro 1).

\section{Procedimiento}

Se realizó un experimento con 26 genotipos de maíz, bajo condiciones de riego en el ciclo primavera-verano, para definir su productividad, bajo el siguiente esquema: 
La siembra se realizó en el mes de abril. Se trazaron parcelas de 5 surcos de $0.90 \mathrm{~cm}$ de ancho x 5 metros de largo, y se depositó una semilla por golpe cada $12 \mathrm{~cm}$, a una profundidad de $7 \mathrm{~cm}$ en forma manual (92,463 semillas/ha). La fertilización se realizó con una dosis de 160-40-00 (N-P-K), aplicando todo el fósforo (P), y la tercera parte del nitrógeno $(\mathrm{N})$ al cultivo a la siembra y las otras dos terceras partes del $\mathrm{N}$; una se aplicó al mes y la otra parte dos meses después de la siembra, respectivamente. El control de malezas se hizo con herbicidas pos-emergentes (Gesaprin $\AA$ ) con una bomba aspersora de mochila manual, 25 días después de la siembra (DDS), una vez que las plántulas de maíz habían emergido (12 a $15 \mathrm{~cm}$ ); y una segunda a los 40 días después, con el mismo producto y de la misma manera.

Cuadro 1. Relación de genotipos evaluados como tratamientos

\begin{tabular}{|c|c|c|}
\hline Genotipo & Origen & Región \\
\hline RazaJala & Maíz blanco de la raza Jala local de los Valles de Jala, Nayarit & Subtropica \\
\hline Tampiqueño1 & Maíz blanco local Tampiqueño de los Valles de Jala, Nayarit & Subtropica \\
\hline Tampiqueño2 & Maíz blanco local Tampiqueño de los Valles de Jala, Nayarit & Subtropical \\
\hline AForrajero & Maíz amarillo local con fines forrajeros en los Valles de Jala, Nayarit & Subtropical \\
\hline AElotero & Maíz amarillo local con fines de elote en los Valles de Jala, Nayarit & Subtropica \\
\hline FcolMadJala & Maíz blanco de la raza Jala introducido a zonas altas de Jala, Nay. & Subtropica \\
\hline SantaFeJala & Maíz blanco local de las zonas altas de Jala, Nayarit & Subtropical \\
\hline HojeroUze & Maíz blanco seleccionado de tercer ciclo, introducido al sur de Nayarit & Subtropical \\
\hline PapantlalntrJala & Maíz blanco-amar. de Papantla, Ver. con interregresión de la raza Jala & Tropical \\
\hline PapantlalntrJala2 & Maíz blanco de Papantla, Veracruz con interregresión de la raza Jala & Tropical \\
\hline PaplntrJalaAmc & Maíz Amarillo claro de Papantla, Ver. con interregresión de la raza Jala & Tropical \\
\hline PapantlalntrJala1 & Maíz blanco de Papantla, Ver. con interregresión de la raza Jala & Tropical \\
\hline OjitallntrPap & Maíz Amarillo de Castillo de Teallo, Ver. Interregresión Papantla, Ver. & Tropical \\
\hline OjitalCT & Maíz blanco local de Castillo de Teallo, Ver. & Tropical \\
\hline BVPbaja & Maíz blanco local de Bellavista, Papantla, Ver. & Trop \\
\hline AS948-2 & Maíz blanco comercial de tercer ciclo & Subtropical \\
\hline TlanchiHgoA1 & Maíz amarillo local de Tlanchinol, Hgo. & Subtropical \\
\hline TlanchiHgoA2 & Maíz amarillo local de Tlanchinol, Hgo. & Subtropical \\
\hline TlanchiHgoA3 & Maíz amarillo local de Tlanchinol, Hgo. & Subtropical \\
\hline TlanchiHgoA4 & Maíz amarillo local de Tlanchinol, Hgo. & Subtropical \\
\hline TlanchiHgoA5 & Maíz amarillo local de Tlanchinol, Hgo. & Subtropical \\
\hline TlanchiHgoA6 & Maíz amarillo local de Tlanchinol, Hgo. & Subtropical \\
\hline TlanchiHgoA7 & Maíz amarillo local de Tlanchinol, Hgo. & Subtropical \\
\hline Forrasierra & Maíz amarillo comercial para Zona Centro de San Luis Potosí & Semiárida \\
\hline Cerritos & Maíz blanco local de Cerritos, San Luis Potosí & Semiárida \\
\hline Mexquitic & Maíz blanco local de Mexquitic, San Luis Potosí & Semiárida \\
\hline
\end{tabular}

El riego fue por gravedad, y se aplicó en promedio cada 21 días después de la emergencia, con una lámina aproximada de $10 \mathrm{~mm}$, y hasta cuando el elote estuvo en el 
estado de $1 / 2$ de la línea de leche. Se marcaron 10 plantas para cada parcela en competencia completa al azar, y se identificaron con una etiqueta para medir las variables evaluadas.

La cosecha se realizó a los 120 a 135 DDS, según como fueron llegando los elotes al 1/2 de la línea de leche de cada respectivo genotipo; para ello las plantas marcadas se cosecharon al ras del suelo, se pesaron con una balanza manual colgante de $20 \mathrm{~kg}$ $\left(\mathrm{GAMO} \AA^{8}\right)$, con una aproximación de $0.5 \mathrm{~kg}$. Para después separar los componentes, tallo+hoja y elote que se pesaron en forma individual, en una báscula digital (TORREY® Modelo EQ-5/10), con una capacidad de $5 \mathrm{~kg}$ y una aproximación de $1 \mathrm{~g}$. Cada componente fue triturado y molido en un molino de forraje verde con cuchillas y martillos (NOGUERA®). Se tomó una muestra del material molido de $200 \mathrm{~g}$ para cada componente, y se depositaron en bolsas de papel estraza, previamente identificadas por genotipo y repetición; las cuales fueron llevadas a una estufa de aire forzado para secarlas durante $120 \mathrm{~h}$ a $55^{\circ} \mathrm{C}$. Una vez secas se pesaron en una balanza digital marca Ohaus, con capacidad de $300 \mathrm{~g}$ y una aproximación de $0.1 \mathrm{~g}$, y se determinó el porcentaje de MS. El porcentaje de MS de cada muestra y de cada componente se multiplicó a la materia verde, calculada por hectárea a partir de las 10 plantas muestreadas.

\section{Variables estudiadas}

De diez plantas en competencia completa cosechadas en verde, una vez pesadas y registradas en la bitácora, se separaron en dos componentes elote y planta completa (tallos + hojas); los elotes y la planta completa se pesaron en verde en una balanza Marca TORREY®, Modelo EQ-5/10, con una capacidad de $5 \mathrm{~kg}$ y una aproximación de $1 \mathrm{~g}$. Se picaron en forma separada en una trituradora de forraje de martillos con navajas, y se tomó una muestra de $200 \mathrm{~g}$ que se colocaron en una bolsa de papel estraza, la cual se llevó a una estufa de secado de aire forzado, para determinar la MS; luego se pesó en una balanza CS200 (Ohaus $\AA$ ), con una aproximación a $0.1 \mathrm{~g}$, de tal forma que se obtuvieron dos variables de componentes:

Rendimiento de materia seca de planta completa (tallo + hoja) (RMSP).

Rendimiento de materia seca de elote (RMSE).

Rendimiento de materia seca (RMS), se obtuvo sumando los dos componentes planta y elote.

Relación elote: planta completa (RELPC). Se obtuvo dividiendo el rendimiento de la MS del elote, entre el rendimiento de la MS de la planta completa (Elotes, Tallo + Hojas).

Relación planta: planta completa (RPPC). El rendimiento de la MS del tallo + hoja se dividió entre el rendimiento de MS de la planta completa.

Número de hojas por planta (NHOJAS). Se contó el número de hojas por cada planta, dato que se registró en forma individual para las 10 plantas con competencia completa tomadas al azar, de la unidad experimental.

Número de elotes por planta (NELOTES). Se contó el número de elotes en las 10 plantas seleccionadas al azar. 
Altura de planta (AP). Se midió con cinta métrica, desde la base del tallo a la base de inserción de la lámina de la hoja con la vaina de la última hoja, en las 10 plantas seleccionadas al azar

Diámetro de tallo (DT). Se midió en el entrenudo inferior con un vernier manual a las diez plantas seleccionadas al azar.

\section{Diseño Experimental}

El diseño experimental fue completamente al azar, con seis repeticiones para RMS y 10 repeticiones para NHOJAS, NELOTES, AP y DT; donde la parcela experimental fue de 5 surcos de $0.90 \mathrm{~m}$ de ancho y $5 \mathrm{~m}$ de largo.

Los datos obtenidos se analizaron mediante el paquete estadístico SAS® Versión 9.3 y se realizó la prueba de Tukey $\mathrm{P}<0.05$ para la comparación de medias (SAS, 2011).

\section{RESULTADOS Y DISCUSIÓN}

\section{Rendimiento de materia seca de planta}

RMSP mostró significancia (cuadro 2), donde OjitalCT y Tampiqueño1 mostraron los mayores valores con 17.8 y $17.0 \mathrm{t} \mathrm{MSP} \mathrm{ha}^{-1}$, a las que les siguieron los genotipos TlanchiHgo2, PapantlalntrJala, TlanchiHgoA7 y AS948-2 con 15.7, 14.6, 14.6 y $14.0 \mathrm{t}$ MSP ha-1, respectivamente. El genotipo con menor valor fue TlanchiHgoA3 con 3.4 t MSP ha $^{-1}$. Valores mayores a los de Elizondo y Boschini (2002), en maíces criollos y mejorados en un bosque húmedo montañoso, con valores de 11.3 y 7.4 t MSTH (MSP) ha-1, respectivamente. Para hoja de 5.7 a $5.0 \mathrm{t} \mathrm{ha}^{-1}$ y para tallo 19.1 a $13.6 \mathrm{t} \mathrm{ha}^{-1}$. Valores menores a los observados por Rivas et al. (2018), en maíces evaluados en trópico seco para hoja de 24.8 a $18.6 \mathrm{t} \mathrm{ha}^{-1}$ de tallo+hojas (MSP). Similares a los obtenidos por Elizondo-Salazar (2011), con 7.8 y 13.8 t de tallo+hojas (MSP) ha-1 para maíz mejorado y criollo, respectivamente.

\section{Rendimiento de materia seca de elote}

RMSE mostró diferencias significativas (cuadro 2), donde los genotipos con los mayores valores fueron TlanchiHgoA2, con 12.7 t MSE ha $^{-1}$ y le siguió FeliUzeta, con 9.0 t de MSE $\mathrm{ha}^{-1}$. El genotipo con el menor valor fue FcolMaJala con $1.2 \mathrm{t} \mathrm{MSE} \mathrm{ha-1}^{-1}$. Valores semejantes a los obtenidos por Rivas et al. (2018) en híbridos trilineales evaluados en trópico seco de 8.9 a $8.5 \mathrm{t} \mathrm{MSE} \mathrm{ha-1}^{-1}$, y menores a los obtenidos por Elizondo y Boschini (2002) en maíces criollos y mejorados en un bosque húmedo montañoso, con valores de 10.7 y 15.3 t MS de elote ha-1 ${ }^{-1}$, respectivamente; y a los de Elizondo-Salazar (2011), con 3.1 y 1.5 t de MSEL ha-1 para maíz mejorado y criollo, respectivamente. Lo que demuestra la alta productividad de los maíces tropicales, semitropicales y templados, en comparación a maíces de montaña. Además de observarse variabilidad de este componente entre los genotipos, que bien este carácter puede aprovecharse para futuro mejoramiento. 


\section{Rendimiento de materia seca}

EI RMS fue significativo entre genotipos (cuadro 2), donde el mayor valor lo obtuvo el genotipo TlanchiHgoA2, con $28.6 \mathrm{t} \mathrm{MS} \mathrm{ha}^{-1}$; a la que le siguió el genotipo OjitalCT, con $25.5 \mathrm{t}$ de $\mathrm{MS} \mathrm{ha}^{-1}$. El menor valor lo mostró el genotipo TlanchiHgoA3, con $8.1 \mathrm{t} \mathrm{MS} \mathrm{ha-1}^{-1}$. Estos resultados son similares a los observados por Zaragoza-Esparza et al. (2019) de 26.4 a 24.3 t ha $^{-1}$ en maíces híbridos. Datos menores a los de Parra (1996) para 23 genotipos de maíz criollo y dos variedades comerciales, con un rango de 18.13 a $33.9 \mathrm{t}$ MS ha-1 para 1991; a los de Rivas et al. (2011) para maíces trilineales, evaluados en Valles Altos con 47.2 a $34.3 \mathrm{t} \mathrm{MS} \mathrm{ha}^{-1}$, y a los de Rivas et al. (2018) para maíces trilineales

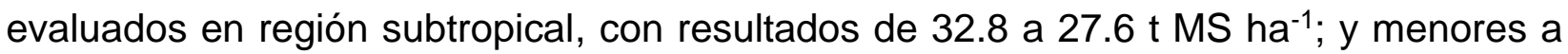

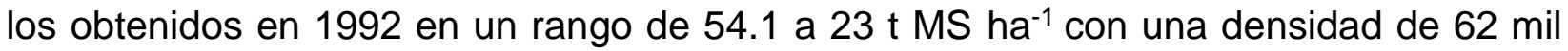
plantas ha-1 ${ }^{-1}$ y mayores a los observados por Sánchez et al. (2011), para maíz criollo, que mostró el mayor rendimiento con 44.2 t de materia verde ha- ${ }^{-1}$; que si consideramos un porcentaje de materia seca de $35 \%$ por el estado en que se cosechó, tendríamos un rendimiento de $15.5 \mathrm{t} \mathrm{MS} \mathrm{ha}^{-1}$ bajo condiciones cálidas húmedas, y a los de Elizondo y Boschini (2002) en maíces criollos con valores de 11.6 t MS ha-1. $^{-1}$

\section{Relación Planta: Planta Completa}

EI RPPC mostró diferencias significativas (cuadro 2), donde el mayor valor lo obtuvo el genotipo FcolMaJal, con 0.91; a la que le siguieron PaplntrJalaAmc, con 0.80 y PapantlalntrJala, Tampiqueño1 y Tampiqueño2, con 0.76 para los tres. Por otro lado, Rivas et al. (2018) observaron valores de hoja de 0.16 a 0.22 y tallo de 0.43 a 0.58 , que sumando en total los dos componentes darían 0.59 a 0.80 de RPPC, un poco menor a lo observado en esta investigación. Rivas et al. (2011) observó otros valores para maíces trilineales, evaluados en Valles Altos con 0.77 a 0.55 .

\section{Relación Elote: Planta Completa}

EI RELPC mostró diferencia estadística entre genotipos (cuadro 2), donde TlanchiHgoA3 mostró el mayor valor con 0.61; seguido del genotipo Forrasierra, con 0.53; mientras que el menor valor lo obtuvo FcolMaJal, con 0.10. Datos mayores a los observados por Rivas et al. (2011) con valores para maíces trilineales evaluados en Valles Altos, con $0.45 \mathrm{a}$ 0.23., y a los de Rivas et al. (2018) con elote 0.26 a 0.35. Este carácter es importante seleccionarlo en los maíces para forraje, porque una proporción superior al $54 \%$ de elotes puede asegurar una digestibilidad in vitro (DIV) mayor a 68\% (Peña et al., 2004) y mostraron menos del $50 \%$ de FDN, por presentar un valor energético satisfactorio (Núñez et al., 2003). 
Cuadro 2. Comparación de medias de Rendimiento de materia seca, de Planta completa (tallo+hoja) y elote, y relaciones de componentes con planta de 26 genotipos. Soledad de Graciano Sánchez, S. L. P.

\begin{tabular}{|c|c|c|c|c|c|}
\hline GENOTIPO & RMSP & RMSE & RMS & RPPC & RELPC \\
\hline PapantlalntrJala1 & 11680 abc & $4555 \mathrm{bc}$ & 16235 abc & $0.73^{b}$ & $0.27 \mathrm{abcd}$ \\
\hline FcolMaJal & $10487 a b c$ & $1198^{c}$ & 11685 bc & 0.91 a & $0.10^{d}$ \\
\hline RazaJala & $12712 a b c$ & 3461 bc & $16173 a b c$ & $0.69 \mathrm{~b}$ & $0.31 \mathrm{abcd}$ \\
\hline Forrasierra & $5547 \mathrm{bc}$ & $6356 a b c$ & 11903 bc & $0.47^{b}$ & $0.53 \mathrm{ab}$ \\
\hline PapIntrJalaAmc & $12462 a b c$ & 3171 bc & 15634 abc & $0.80 a b$ & $0.21 \mathrm{~cd}$ \\
\hline PapantlalntrJala2 & $10020 a b c$ & $3208 b c$ & 13227 bc & $0.76 a b$ & $0.24 \mathrm{bcd}$ \\
\hline TlanchiHgoA6 & $10842 a b c$ & 5334 bc & 16176 abc & $0.68 \mathrm{~b}$ & 0.32 abcd \\
\hline AS948-2 & $14017 \mathrm{ab}$ & $7064 a b c$ & 21081 abc & $0.67 \mathrm{~b}$ & $0.33 \mathrm{abcd}$ \\
\hline OjitallntrPap & $9751 \mathrm{abc}$ & 4356 bc & $14107 \mathrm{abc}$ & $0.70^{b}$ & $0.30 \mathrm{abcd}$ \\
\hline PapantlalntrJala & $14623 a b$ & $5923 a b c$ & $20546 a b c$ & $0.72 b$ & $0.29 \mathrm{abcd}$ \\
\hline TlanchiHgoA7 & $14596 \mathrm{ab}$ & $5958 a b c$ & $20553 a b c$ & $0.72 \mathrm{~b}$ & 0.29 abcd \\
\hline TlanchiHgoA4 & 11768 abc & $6845 a b c$ & $18613 a b c$ & $0.63 \mathrm{~b}$ & $0.37 \mathrm{abcd}$ \\
\hline HojeroUze & $11100 \mathrm{abc}$ & $8959 a b$ & $20063 a b c$ & $0.56 \mathrm{~b}$ & $0.44 a b c$ \\
\hline Cerritos & $13249 a b c$ & $7561 \mathrm{abc}$ & $20810 a b c$ & $0.65 \mathrm{~b}$ & $0.35 \mathrm{abcd}$ \\
\hline Tampiqueño1 & 17031 a & $6164 a b c$ & $23195 a b c$ & $0.76 \mathrm{ab}$ & 0.24 bcd \\
\hline TlanchiHgoA1 & $11415 a b c$ & $6769 a b c$ & $18184 a b c$ & $0.63 \mathrm{~b}$ & $0.38 \mathrm{abcd}$ \\
\hline TlanchiHgoA5 & $10153 a b c$ & 3764 bc & $13917 \mathrm{abc}$ & $0.72 \mathrm{~b}$ & 0.29 abcd \\
\hline TlanchiHgoA2 & $15863 \mathrm{ab}$ & 12712 a & 28582 a & $0.56 \mathrm{~b}$ & $0.44 \mathrm{abcd}$ \\
\hline Tampiqueño2 & 11920 abc & $3823 b c$ & $15743 a b c$ & $0.76 \mathrm{ab}$ & $0.24 \mathrm{bcd}$ \\
\hline AForrajero & $9228 a b c$ & $7036 a b c$ & $16263 a b c$ & $0.57 \mathrm{~b}$ & 0.43 abcd \\
\hline AElotero & $10749 a b c$ & $5459 a b c$ & 10787 bc & $0.63 \mathrm{~b}$ & 0.38 abcd \\
\hline TlanchiHgoA3 & $3427^{c}$ & $4621 a b c$ & $8047^{c}$ & $0.39 \mathrm{~b}$ & 0.61 a \\
\hline SantaFeJala & $10895 a b c$ & $7305 a b c$ & $18199 a b c$ & $0.65 \mathrm{~b}$ & 0.35 abcd \\
\hline OjitalCT & 17822 a & $7655 a b c$ & $25477 a b$ & $0.70^{b}$ & $0.30 \mathrm{abcd}$ \\
\hline BVPbaja & $11313 a b c$ & $4705 \mathrm{bc}$ & $16018 a b c$ & $0.71 \mathrm{~b}$ & 0.29 abcd \\
\hline Mexquitic & $9999 a b c$ & $5998 a b c$ & $15997 \mathrm{abc}$ & $0.62 \mathrm{~b}$ & $0.39 \mathrm{abcd}$ \\
\hline Media & 11641 & 5768 & 17200 & 0.69 & 0.34 \\
\hline DMS & 10522 & 7328 & 15161 & 0.55 & 0.38 \\
\hline
\end{tabular}

${ }^{*}$ Letras $a, b, c, \ldots$ diferentes por columna muestras diferencias significativas. RMSP=Rendimiento de materia seca de la planta (tallo+hojas). RMSE=Rendimiento de materia seca de elote. RMS=Rendimiento de materia seca. RPPC=Relación planta:planta completa. RELPC=Relación elote:planta completa. DMS=Diferencia mínima significativa.

\section{Altura}

Para la altura, los resultados mostraron diferencias significativas entre genotipos (cuadro 3), donde Tampiqueño1 mostró el mayor valor con $241.8 \mathrm{~cm}$, y le siguió el genotipo, PapantlalntrJala2 con $231.9 \mathrm{~cm}$; en cambio el híbrido comercial Forrasierra mostró el menor valor con $135.8 \mathrm{~cm}$. Los datos mostraron gran variabilidad en la altura de la planta. Estos resultados son semejantes a los encontrados por Parra (1996), quien observó para 23 genotipos de maíz criollo un rango de altura de 245 a $169 \mathrm{~cm}$. en 1991, y 239 a 175 cm. en 1992; al igual que a los observados por Sánchez et al. (2011), para maíz criollo con 244 y $216 \mathrm{~cm}$ de altura, de una región cálida húmeda; también a los de Sánchez et al. (2013), quienes obtuvieron alturas de 247 y $216 \mathrm{~cm}$ para un criollo y de 195 a $165 \mathrm{~cm}$ 
Cuadro 3. Comparación de medias de Altura de planta, Diámetro de tallo y Número de hojas de 26 genotipos de maíz. Soledad de Graciano Sánchez, S. L. P.

\begin{tabular}{|c|c|c|c|}
\hline GENOTIPO & $\begin{array}{l}\text { ALTURA DE PLANTA } \\
\text { (cm) }\end{array}$ & $\begin{array}{l}\text { DIAMETRO DE } \\
\text { TALLO (cm) }\end{array}$ & $\begin{array}{l}\text { NUMERO DE } \\
\text { HOJAS }\end{array}$ \\
\hline PapantlalntrJala1 & 205.8 bcdefgh & $2.74 \mathrm{abcd}$ & 16.3 bcdefg \\
\hline FcolMaJal & 211.3 abcdef & 2.59 abcdef & 16.5 bcdef \\
\hline RazaJala & $217.9 a b c$ & 2.96 a & 19.0 a \\
\hline Forrasierra & 135.8 i & $2.14^{h}$ & $12.4^{\mathrm{i}}$ \\
\hline PapIntrJalaAmc & 214.0 abcde & 2.57 abcdef & 17.5 abcd \\
\hline PapantlalntrJala2 & $231.9 a b$ & $2.82 a b c$ & 17.0 abcde \\
\hline TlanchiHgoA6 & 198.1 bcdefgh & 2.59 abcdef & 15.5 defgh \\
\hline AS948-2 & 213.2 abcdef & $2.87 a b$ & $17.8 \mathrm{abc}$ \\
\hline OjitallntrPap & $220.3 a b c$ & $2.84 a b c$ & $17.3 \mathrm{abcd}$ \\
\hline PapantlalntrJala & 196.3 cdefgh & $2.75 \mathrm{abcd}$ & 15.8 bcdefg \\
\hline TlanchiHgoA7 & 199.7 bcdefgh & 2.57 abcdef & 15.4 defgh \\
\hline TlanchiHgoA4 & 211.2 abcdef & 2.56 abcdefg & $17.7 \mathrm{abcd}$ \\
\hline HojeroUze & 192.1 cdefgh & 2.30 efg & 17.2 abcde \\
\hline Cerritos & 182.3 defgh & 2.36 defg & 15.0 efgh \\
\hline Tampiqueño1 & 241.8 a & 2.57 abcdef & 17.3 abcde \\
\hline TlanchiHgoA1 & 212.1 abcdef & 2.68 abcde & 16.3 bcdefg \\
\hline TlanchiHgoA5 & $179.8 \mathrm{fgh}$ & 2.44 cdefg & 16.5 bcdef \\
\hline TlanchiHgoA2 & 209.2 abcdefg & 2.68 abcde & 17.0 abcde \\
\hline Tampiqueño2 & 215.8 abcd & 2.43 cdefg & 17.2 abcde \\
\hline AForrajero & $173.0^{\mathrm{h}}$ & 2.29 efg & 15.6 cdefgh \\
\hline AElotero & 181.4 efgh & $2.24 \mathrm{fg}$ & $14.1 \mathrm{ghi}$ \\
\hline TlanchiHgoA3 & 208.2 abcdefg & 2.33 defg & 14.4 fghi \\
\hline SantaFeJala & 204.4 bcdefgh & 2.43 defg & 15.5 dfegh \\
\hline OjitalCT & 200.0 bcdefgh & 2.52 bcdefg & 17.2 abcde \\
\hline BVPbaja & 209.0 bcdefg & 2.33 defg & 18.0 ab \\
\hline Mexquitic & $175.3 \mathrm{gh}$ & $2.22 \mathrm{gh}$ & $13.5 \mathrm{hi}$ \\
\hline Media & 201.5 & 2.53 & 16.3 \\
\hline DMS & 34.0 & 0.43 & 2.32 \\
\hline
\end{tabular}

${ }^{*}$ Letras $a, b, c, \ldots$ diferentes por columna muestras diferencias significativas. DMS=Diferencia mínima significativa.

en híbridos comerciales, en una región tropical húmeda en dos experimentos. Esta altura es semejante ya que los criollos que mostraron una mayor altura en esta investigación provienen de regiones cálidas y semicálidas; al igual a los observados por ZaragozaEsparza et al. (2019), con valores de 2.4, 2.3, 2.2, 2.2 y $2.2 \mathrm{~m}$ para los maíces de mayor porte en Valles Altos. Mayores a los observados por Sánchez et al. (2019), para maíces tropicales, con valores de 203.1 a $167.2 \mathrm{~cm}$. Menores a los observados Rivas et al. (2011), quienes obtuvieron valores para maíces trilineales evaluados en Valles Altos de 284 a $201 \mathrm{~cm}$; y a los de Rivas et al. (2018), en maíces evaluados en trópico seco, con un rango de 264 a $195 \mathrm{~cm}$; Yescas et al. (2015), con valores que van de 257 a $282 \mathrm{~cm}$; Borroel et al. (2014), con 243 a $221 \mathrm{~cm}$; Montemayor et al. (2006) con rango de 172 a $156 \mathrm{~cm}$ y Rivas et al. (2006) de 255 a $159 \mathrm{~cm}$.

\section{Diámetro de tallo}

El Diámetro de tallo mostró significancia estadística (cuadro 3), donde el genotipo RazaJala mostró el mayor valor con $2.96 \mathrm{~cm}$, a la que le siguió AS948-2 con $2.87 \mathrm{~cm}$; en 
tanto que el híbrido comercial Forrasierra mostró el menor valor con $2.14 \mathrm{~cm}$. De tal forma que se observó una gran variabilidad entre genotipos para el DT, siendo un carácter que puede utilizarse para selección y mejoramiento genético. Datos mayores a los observados por Parra (1996) para 23 genotipos de maíz criollo, en un rango de 2.01 a $1.28 \mathrm{~cm}$ en 1991 y de 1.93 a $1.36 \mathrm{~cm}$ en 1992; y semejantes a los observados por Rivas et al. (2011) para maíces trilineales evaluados en Valles Altos con 2.71 a $2.30 \mathrm{~cm}$.

\section{Número de hojas}

NHOJAS mostró diferencias significativas (cuadro 2), donde el genotipo con mayor valor fue RazaJala con 19.0 hojas planta $^{1}$, a la que le siguió BVPbaja con 18.0 hojas planta ${ }^{1}$; mientras que el híbrido comercial Forrasierra presentó el menor valor con 12.4 hojas planta ${ }^{1}$. Valores similares a los de Rivas et al. (2018) con 17.0 a 14.2 en maíces trilineales evaluados en trópico seco. Datos mayores a los obtenidos por Parra (1996) en 21 criollos, al obtener un rango de 16.4 a 14.5 hojas por planta, y muchos mayores a los de Sánchez et al. (2019) con valores de 10.7 a 9.6 hojas planta $^{-1}$, y similares a los de Rivas et al. (2011) maíces trilineales evaluados en Valles Altos con 14.9 a 12.5. Este carácter es importante seleccionarlo en los maíces porque es uno de los componentes que le confieren mayor valor nutritivo a los genotipos, por ser de mayor digestibilidad (Paliwal, 2001).

\section{CONCLUSIÓN}

Con respecto al número de hojas, los genotipos RazaJala, BVPbaja y AS948-2 con valores que van en un rango de 19 a 17.8 hojas, mostraron los valores más altos, lo que los hace recomendables para seleccionarlos o recomendarlos por este rango considerable. En cuanto rendimiento de materia seca total, los mejores genotipos fueron TlanchiHgoA2 y OjitalCT, demostrando características productivas que pueden ser tomadas en cuenta para la producción de materia seca. Para el rendimiento de materia seca planta (tallo + hoja), OjitalCT y Tampiqueño1 demostraron tener el mayor valor, lo que hace que estos genotipos sean viables para producción de materia seca en tallo y hoja; asimismo para un buen rendimiento. De acuerdo a la evaluación, se observó que existen genotipos de región subtropical y tropical, que muestran un comportamiento productivo satisfactorio y mejor que otros locales e híbridos mejorados en regiones semiáridas, como el Tampiqueño1, OjitalCT y TlanchiHgoA2; por lo que podrían recomendarse para ser evaluados más ampliamente a nivel comercial para ser utilizados como alternativas de maíz forrajeros, o considerarlos en programas de mejoramiento para fines forrajeros.

\section{LITERATURA CITADA}

BORROEL GVJ, Álvarez RVP, Rodríguez HSA, Jiménez DF, Preciado RP, Ogaz A, Zermeño GH. 2014. Rendimiento de maíz forrajero bajo la adición de ácido húmico y 
algaenzima. Revista Iberoamericana de Ciencias. 1(2):233-244. http://www.reibci.org/publicados/2014/julio/2200131.pdf.

ELIZONDO-SALAZAR JA. 2011. Influencia de la variedad y altura de cosecha sobre el rendimiento y valor nutritivo de maíz para ensilaje. Agronomía Costarricense. 35(2):105111. https://www.mag.go.cr/rev_agr/v35n02_105.pdf.

ELIZONDO J, Boschini C. 2002. Producción de forraje con maíz criollo y maíz híbrido. Agronomía Mesoamericana. 13:13-17. http://www.mag.go.cr/rev_meso/v13n01_013.pdf.

GARCÍA AE. 2004. Modificaciones al sistema de clasificación climática de Koppen. Quinta Edición. Instituto Nacional de Geografía. UNAM. México, D. F. Pp. 246. ISBN 9703210104.

GONZÁLEZ CF, Peña RA, Núñez HG, Jiménez GC. 2005. Efecto de la densidad y altura de corte en el rendimiento y calidad del forraje de maíz. Rev. Fitotec. Mex. 28(4):393-397. https://www.revistafitotecniamexicana.org/documentos/28-4/13a.pdf.

GONZÁLEZ-SALAS U, Gallegos-Robles MA, Vázquez-Vázquez C, García-Hernández JL, Fortis-Hernández M, Mendoza-Retana SS. 2018. Productividad de genotipos de maíz forrajero bajo fertilización orgánica y propiedades físico-químicas del suelo. Revista Mexicana de Ciencias Agrícolas. 20(1):4331-4341. https://doi.org/10.29312/remexca.v0i20.1002.

MONTEMAYOR TJA, Zermeño GA, Olague RJ, Aldaco NR, Fortis HM, Salazar SE, Cruz RJC, Vázquez-Vázquez C. 2006. Efecto de la densidad y estructura del dosel de maíz en la penetración de la radiación solar. Phyton. 55(75): 47-53. http://www.revistaphyton.fund-romuloraggio.org.ar/vol75.html.

NÚÑEZ HG, Faz CR, González CF, Peña RA. 2005. Madurez de híbridos de maíz a la cosecha para mejorar la producción y calidad del forraje. Téc. Pec. Méx. 43(1):69-78. https://dialnet.unirioja.es/servlet/articulo?codigo $=1103930$.

NÚÑEZ HG, Contreras GEF, Faz CR. 2003. Características agronómicas y químicas importantes en híbridos de maíz para forraje con alto valor energético. Técnica Pecuaria en México. 41:37-48.

https://pdfs.semanticscholar.org/1e51/4fd5c4accabc935a88d0634d4a7b6598b04a.pdf.

PALIWAL RL. 2001. Mejoramiento del maíz con objetivos especiales. In: Paliwal RL, Granados G, Lafitte HR, Violic AD. (eds). El maíz en los trópicos. Mejoramiento y producción. Departamento Agrícola. FAO. Roma, Italia. http://www.fao.org/3/x7650s00.htm.

PARRA A. 1996. Evaluación de cultivares criollos e híbridos de maíz (Zea mays L.) para uso forrajero bajo condiciones de bosque seco tropical. Rev. Fac. Agron. (LUZ). 13:251260 . 
https://scholar.google.com.mx/scholar?q=Rev.+Fac.+Agron.+(LUZ).+13:251:260.\&hl=es \&as_sdt=0\&as_vis $=1 \&$ oi $=$ scholart

PEÑA RA, González CF, Núñez HG, Jiménez GC. 2004. Aptitud combinatoria de líneas de maíz para alta producción y calidad forrajera. Revista Fitotecnia Mexicana. 27 (Especial1):1-6. https://www.revistafitotecniamexicana.org/documentos/271\%20Especial\%201/1a.pdf.

RIVAS JMA, Carballo CA, Quero CAR, Hernández GA, García SG, Vaquera HH. 2011. Evaluación productiva y forrajera de doce híbridos de maíz bajo labranza mínima en valles altos. En: Desarrollo de la Agricultura Sostenible. Alternativas Tecnológicas y Enfoques Sociales. Eds: Ramón Díaz-Ruiz, Jesús Felipe Álvarez-Gaxiola, Arturo Huertade la Peña. Editorial Altres Costa-Amic Editores, S. A. de C. V. Puebla, Pue., México. Pp. 129-141. ISBN: 978-968-839-580-6, 978-607-8154-04-3.

RIVAS JMA, Carballo CA, Quero CAR, Hernández GA, Vaquera HH, Rivas ZEC, Rivas ZMA, Rivas ZEJ. 2018. Comportamiento productivo de doce híbridos trilineales de maíz para forraje en una región tropical seca. Tropical and Subtropical Agroecosystems. 21(3):579-586. E-ISSN 1870-0462.

https://pdfs.semanticscholar.org/7727/0b535982ba1cc1a5fc6b38ec1ad6c98763f9.pdf.

RIVAS-JACOBO MA, Carballo-Carballo A, Quero-Carrillo AR, Mendoza-Pedroza SI, Vaquera-Huerta H, Rivas-Zarco MA, Sánchez-Hernández MA. 2019. Productividad y caracteres morfológicos de híbridos de maíz forrajero. Agroproductividad. 12 (8):59-65. ISSN 25940252. https://doi.org/10.32854/agrop.v0i0.1452.

RIVAS JMA, Carballo CA, Pérez PJ, González JG, García ZA. 2006. Rendimiento y calidad de ensilado de seis genotipos de maíz cosechados en dos estados de madurez. En: INIFAP, UV, CP, UACH, ITUG, ITBOCA y UNAM. Avances en la Investigación Agrícola, Pecuaria, Forestal y Acuícola en el Trópico Mexicano. Libro Científico No. 3. Veracruz, México. Primera Edición. Editorial Atlántida Casa de Ciencia y Cultura, SA de CV. Pp. 313-320. ISBN 970-43-0068-9.

SÁNCHEZ MA, Aguilar CU, Valenzuela N, Joaquín BM, Sánchez C, Jiménez MC, Villanueva C. 2013. Rendimiento en forraje de maíces del trópico húmedo de México en respuesta a densidades de siembra. Rev.Mex.Cienc.Pec. 4(3):271-288. ISSN: 24486698.

https://cienciaspecuarias.inifap.gob.mx/index.php/Pecuarias/article/view/3188/2613.

SÁNCHEZ MA, Aguilar CU, Valenzuela N, Sánchez C, Jiménez MC, Villanueva C. 2011. Densidad de siembra y crecimiento de maíces forrajeros. Agron. Mesoam. 22(2):281295. https://www.scielo.sa.cr/pdf/am/v22n2/a05v22n2.pdf.

SÁNCHEZ HMA, Cruz VM, Sánchez HC, Morales TG, Rivas JMA, Villanueva VC. 2019. Rendimiento forrajero de maíces (Zea mays L.) adaptados al trópico húmedo de México. 
Revista Mexicana de Ciencias Agrícolas. 10(3):699-712. https://dialnet.unirioja.es/servlet/articulo?codigo=6920844.

SAS Institute Inc. 2011. Base SAS® 9.3. Procedures Guide. Cary, NC; SAS Institute Inc. ISBN 978-1-60764-895-6.

SIAP. SERVICIO DE INFORMACIÓN AGROALIMENTARIA Y PESQUERA. 2020a. Bovinos leche. Población ganadera 2009-2018 cabezas. Secretaría de Agricultura y Ganadería.

https://www.gob.mx/cms/uploads/attachment/file/516351/Inventario_2018_Bovino_leche .pdf

SIAP. SERVICIO DE INFORMACIÓN AGROALIMENTARIA Y PESQUERA. 2020b. Producción agrícola 2018. Secretaría de Agricultura y Ganadería. México.

http://infosiap.siap.gob.mx:8080/agricola_siap_gobmx/AvanceNacionalSinPrograma.do

YESCAS CP, Segura CMA, Martínez CL, Álvarez RVP, Montemayor TJA, Orozco VJA, Frías RJE. 2015. Rendimiento y calidad de maíz forrajero (Zea mays L.) con diferentes niveles de riego por goteo subsuperficial y densidad de plantas. Phyton. 84:272-279. http://www.revistaphyton.fund-romuloraggio.org.ar/vol84-2/Yescas.pdf

ZARAGOZA-ESPARZA J, Tadeo-Robledo M, Espinosa-Calderón A, López-López C, García-Espinosa JC, Zamudio-González B, Turrent-Fernández A, Rosado-Núñez F. 2019. Rendimiento y calidad de forraje de híbridos de maíz en Valles Altos de México. Revista Mexicana de Ciencias Agrícolas. 10(1):101-111. https://cienciasagricolas.inifap.gob.mx/editorial/index.php/agricolas/article/view/1403/18 63. 